\title{
Malignant intercostal psammomatous melanotic schwannoma in a patient with Carney complex
}

\author{
Yea Eun Kang ${ }^{1}$, Jin-Ok Jeong ${ }^{1}$, Kyung-Hee Kim ${ }^{2,3}$, Chang-Seok $\mathrm{Ki}^{4}$, and Hyun Jin $\mathrm{Kim}^{1}$
}

\begin{abstract}
Departments of ${ }^{1}$ Internal Medicine, ${ }^{2}$ Pathology, ${ }^{3}$ Cancer Research Institute, Chungnam National University School of Medicine, Daejeon; ${ }^{4}$ Department of Laboratory Medicine \& Genetics, Samsung Medical Center, Sungkyunkwan University School of Medicine, Seoul, Korea
\end{abstract}

Received: March 15, 2017

Revised : May 30, 2017

Accepted: May 30, 2017

\section{Correspondence to}

Hyun Jin Kim, M.D.

Tel: +82-42-280-7151

Fax: +82-42-280-7995

E-mail: kimhj43@cnuh.co.kr
A 40-year-old woman who experienced progressive right abdominal pain for 1 month was admitted to our hospital. The patient and her family members were diagnosed with Carney complex (CNC). Two years prior to admission, the patient underwent percutaneous needle biopsy of a newly diagnosed intercostal mass near segment $\mathrm{V}$ of the liver by computed tomography (CT) (Fig. 1A) and biopsy suggested the presence of psammomatous melanotic schwannoma (PMS). At admission, CT images showed a $3.9 \times 1.5 \mathrm{~cm}$ mass with ill-defined (Fig. 1B). The size of the mass increased by $1.9 \mathrm{~cm}$ in 2 years, and newly occurring calcification was present. Laparoscopic tumor resection was performed. The tumor biopsy revealed a distinct transition between the hypercellular epithelioid central and hypocellular spindle peripheral zones (Fig. 1 C), together with the melanin-containing spindle cells and psammoma bodies, stained positively for HMB45 (Fig. 1D). Based on positive staining for $\mathrm{S}-100$ protein (Fig. $1 \mathrm{E}$ ), and vimentin, the tumor was diagnosed as a malignant PMS. The 2-[fluorine-18]-fluoro-2-deoxy-D-glu-
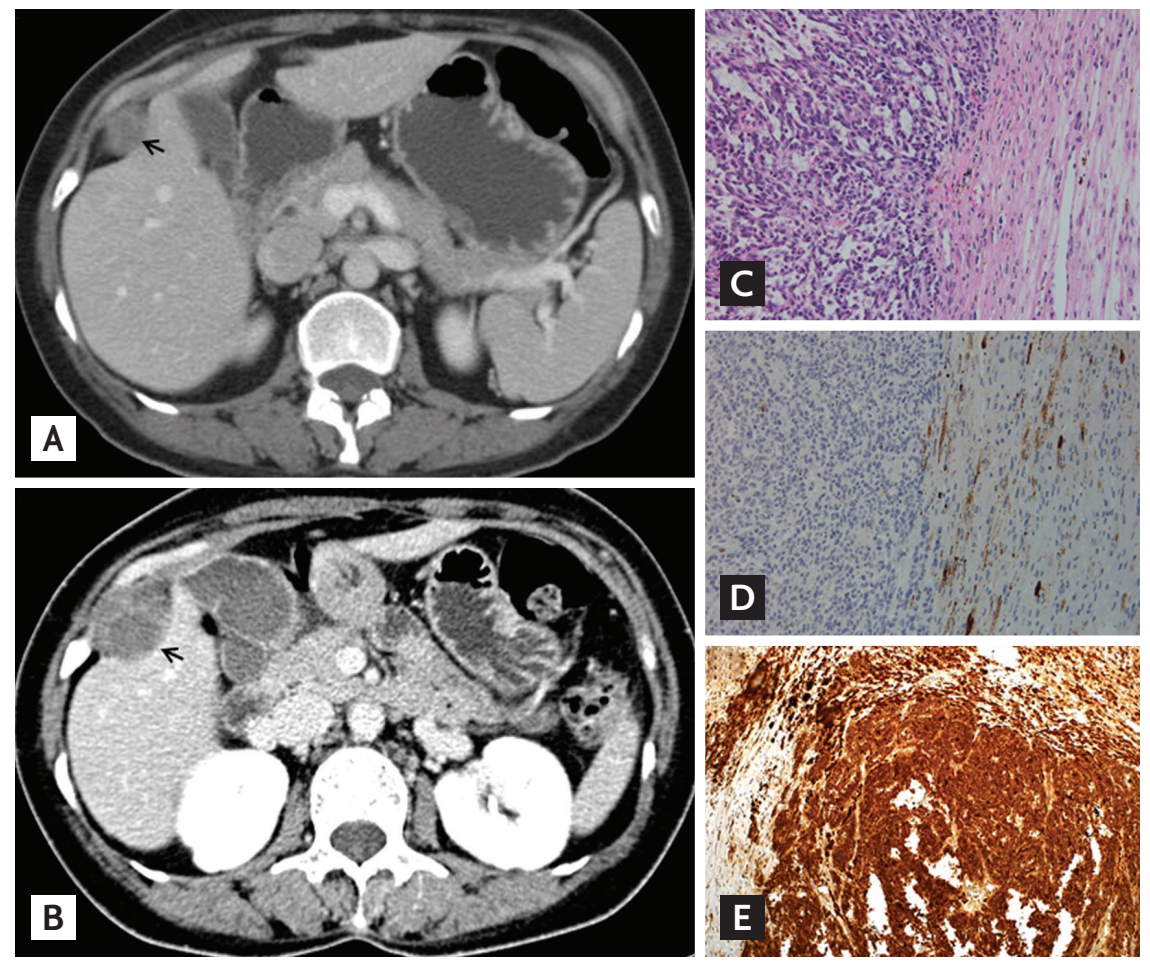
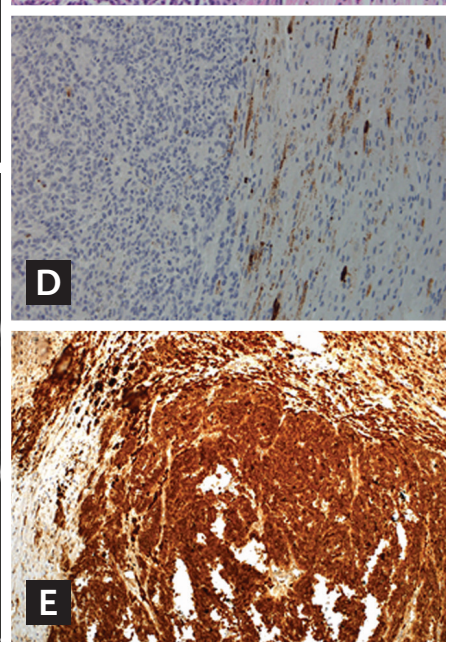

Figure 1. Changes in the size of the intercostal mass on contrast-enhanced abdominal/pelvic computed tomography (CT) and pathologic findings of the intercostal mass. (A) At the initial diagnosis of psammomatous melanotic schwannoma, 2 years prior to admission, a $2.5-\mathrm{cm}$-sized hypoechoic nodule on abdominal/pelvic $\mathrm{CT}$ was revealed next to the $\mathrm{V}$ segment of the liver. (B) At admission, a 4.0-cm-sized, irregularly shaped, hypoechoic nodule with calcification was revealed on abdominal/pelvic CT. All arrows indicated the intercostal mass. (C) The malignant melanotic schwannoma showing a distinct transition between the hypercellular epithelioid central and hypocellular spindle peripheral zones was stained with hematoxylin and eosin (×200). (D) The melanin-containing spindle cells bodies stained positively for $\mathrm{HMB}_{45}$ (×200). (E) The tumor cells were strongly immunopositive for S-10o protein. 

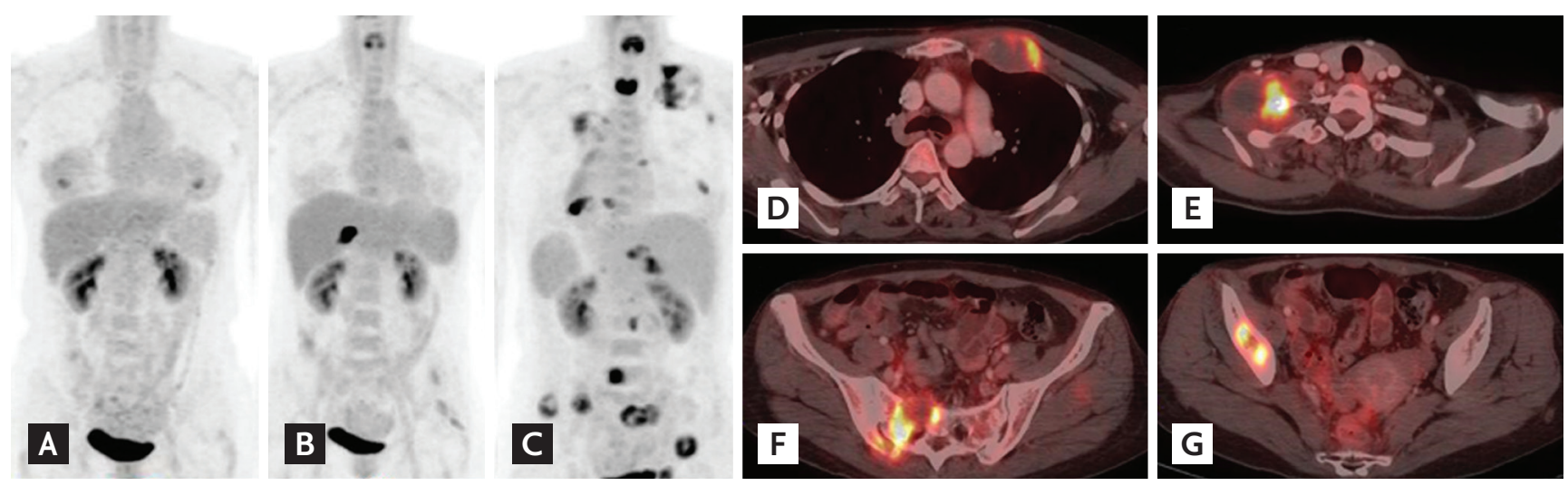

Figure 2. Changes on 2-[fluorine-18]-fluoro-2-deoxy-D-glucose-positon emission tomography/computed tomography (FDGPET/CT) images according to disease progression. (A) At the initial diagnosis of psammomatous melanotic schwannoma, 2 years prior to admission, there was no significant glucose uptake on PET-CT. (B) At admission, PET/CT images revealed intense FDG uptake in the intercostal mass, but without distant metastasis. (C, D, E, F, G) After resection of the tumor, follow-up PET-CT images showed an aggravated state with multiple malignant masses in both lungs, right pleura, bones, and soft tissue.

cose-positon emission tomography/CT (FDG-PET/CT) performed at the initial diagnosis of PMS, showed no significant FDG uptake (Fig. 2A); however, at admission, intense FDG uptake in the intercostal mass was observed (Fig. 2B). Approximately 3 years after the diagnosis of PMS, the patient suffered from tumor growth and distant metastasis (Fig. $2 \mathrm{C}-2 \mathrm{G}$ ), and died despite adjuvant chemotherapy and radiotherapy.

The PMS tumors has been described in isolated cases or, more frequently, in cases of CNC. Although malignant PMS of the spine has been described in patients with CNC, malignant intercostal PMS in CNC has not been reported in the literature to date, to the best our knowledge. Based on our case, we suggest that early complete resection of PMS and rigorous follow-up are necessary for patients with CNC due to the risk of malignant transformation.

\section{Conflict of interest}

No potential conflict of interest relevant to this article was reported. 